Musées, Patrimoine et Culture scientifiques et techniques

$113 \mid 2007$

septembre - octobre 2007

\title{
« Ni vu, ni connu », une scénographie de camouflages
}

\section{Christian Sermet et Martine Millet}

\section{OpenEdition \\ Journals}

Édition électronique

URL : http://journals.openedition.org/ocim/710

DOI : $10.4000 /$ ocim. 710

ISSN : 2108-646X

\section{Éditeur}

OCIM

Édition imprimée

Date de publication : 1 septembre 2007

Pagination : 4-10

ISSN : 0994-1908

\section{Référence électronique}

Christian Sermet et Martine Millet, « « Ni vu, ni connu », une scénographie de camouflages », La Lettre de l'OCIM [En ligne], 113 | 2007, mis en ligne le 09 février 2011, consulté le 19 avril 2019. URL : http:// journals.openedition.org/ocim/710; DOI : 10.4000/ocim.710 


\title{
"Ni vu, ni connu ", une scénographie de camouflages comme média intégré du contenu
}

\author{
Christian Sermet et Martine Millet *
}

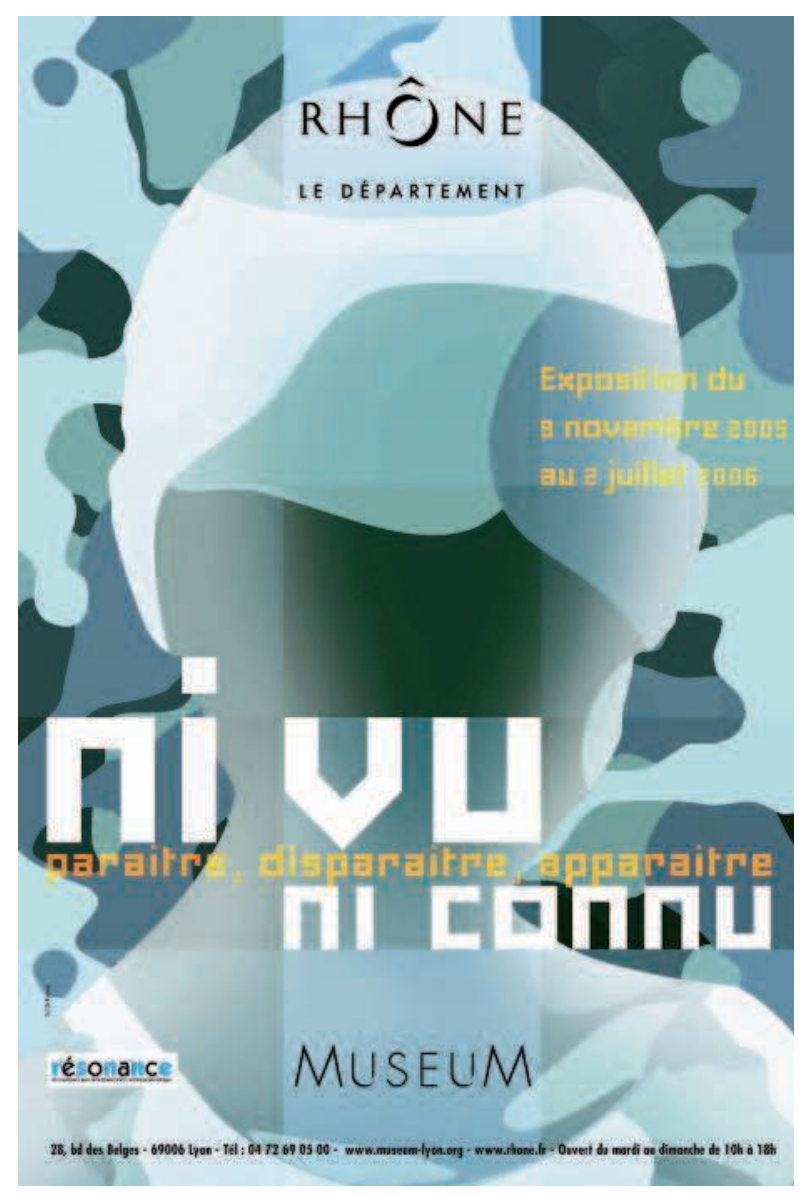

C) Muséum-Département du Rhône

* Christian Sermet, est chargé de projets d'exposition au muséum - musée des Confluences, Lyon christian.sermet@rhone.fr

Martine Millet est responsable du service des expositions au muséum - musée des Confluences, Lyon martine.millet@rhone.fr
Présentée à Lyon au muséum - musée des Confluences au début 2006, l'exposition Ni vu, ni connu a servi de cadre à une expérimentation technologique portant sur l'observation du comportement des visiteurs : les concepteurs mettent ici en évidence les partis pris de conception de l'exposition en matière de choix muséologiques - mise en espace du discours, des objets, des oeuvres, des dispositifs, des textes - et soulignent les partis pris scénographiques, c'est-à-dire le projet muséographique.

«Les hommes en général jugent plus par les yeux que par les mains ; car il échoit à chacun de voir, à peu de gens de percevoir. Chacun voit ce que tu parais, peu perçoivent ce que tu es ». Machiavel, Le Prince

Cet article présente le contexte particulier de réalisation de l'exposition Ni vu, ni connu. Paraître, apparaître, disparaître (1) qui est à l'origine du développement d'une expérience interactive singulière au moyen de puces d'identification automatique par radiofréquence (RFID). Les aspects techniques et statistiques puis les éléments de perception auprès du visiteur de cette expérience menée à l'aide de puces RFID sont présentés dans les deux articles qui suivent. 
Plusieurs partenaires ont collaboré à ce projet : F. Jambon, N. Mandran et C. Perrot (laboratoire d'Informatique de Grenoble, ex CLIPS-IMAG), équipe MultiCom); A.-M. Benoit (CNRS, chargée de recherches en Droit public, chargée des problèmes juridiques relatifs au traitement de l'information et membre de l'intergroupe « Statistiques et Recherche » chargé de la transposition de la Directive européenne d'octobre 1995 sur les données personnelles) ; F. Forest (maison des Sciences de l'Homme - Alpes, Grenoble) ; Y.-A. Martin (centre Érasme, service du département du Rhône en charge de l'innovation numérique), la Commission nationale de l'Informatique et des Libertés, et les équipes du muséum.

C'est dans le cadre du projet du musée des Confluences et d'une phase de préfiguration que le muséum du département du Rhône, à Lyon, développe dans ses expositions, depuis 2000, des expériences muséologiques centrées sur les publics. S’inscrivant dans le caractère « Sciences et Sociétés » du musée des Confluences, l'exposition $\mathrm{Ni} v u$, ni connu développe un traitement thématique et une approche pluridisciplinaire. Ainsi, elle vient en continuité d'expositions thématiques déjà réalisées au muséum telles que Lunes, Chefs-d'œuvre, Trésors et quoi encore..., Blanc comme neige, Sable... En abordant le camouflage comme une notion intimement liée à la personne humaine, cette exposition en fait un véritable sujet de société d'actualité.

\section{Une muséologie de point de vue}

Paraître, apparaître ou disparaître, se montrer, se déguiser ou se cacher... Spontanément associé aux mondes végétal et animal ou à l'univers militaire, avec des fonctions défensives ou offensives, le camouflage, notion qui nous semble familière, ne se limite pourtant pas à ces deux sphères et imprègne aussi fortement les relations humaines. Ni vu, ni connu mobilise donc l'ensemble des connaissances sur le sujet, toutes disciplines confondues : le camouflage dans le domaine des Sciences de la Vie, dans celui des Sciences humaines et plus particulièrement dans celui des Sciences sociales. L'exposition en propose une exploration étendue jusqu'au domaine du rêve et de l'invisibilité sociale.

Depuis quelques années, on constate que la société civile s'approprie et réinvestit les mots et les signes du monde militaire sur le terrain, entre autre, de l'apparence et de la séduction. Qu'est-ce qui a changé pour que la tenue de camouflage et tous ses dérivés soient adoptés aujourd'hui très largement, au-delà de quelques groupes réfractaires ou non à l'idéologie militariste? Nos sociétés occidentales contemporaines qui exploitent donc le camouflage dans la mode, l'art, la télé-réalité ou la publicité, prônent paradoxalement la transparence. Ce véritable phénomène de société, loin d'être marginal et anodin, est porteur de sens, mais que nous dit-il sur nos sociétés actuelles et sur nous-mêmes?

L'homme contemporain oscille entre mise à nu et dissimulation, se donner à voir et se cacher... Voici toute l'ambivalence de l'être humain qui navigue entre une fascination générale pour la chose cachée, l'envie de savoir ce que dissimuleraient les autres et le besoin de conserver son jardin secret.

Quelles sont alors les fonctions sociales de ces camouflages? Qu'en est-il en d'autres temps et dans d'autres cultures ? Peut-on et doit-on tout dire et tout montrer ? Que cache vraiment cette apparente transparence dans nos sociétés du spectacle, de l'information en direct et des reality-shows ? La véritable invisibilité, aujourd'hui, ne serait-elle pas une invisibilité sociale, celle des laissés pour compte?

Autant de questions suscitées au long d'un parcours qui progresse des camouflages les plus familiers, voire stéréotypés, vers des questionnements sur d'autres types de camouflages, moins couramment envisagés.

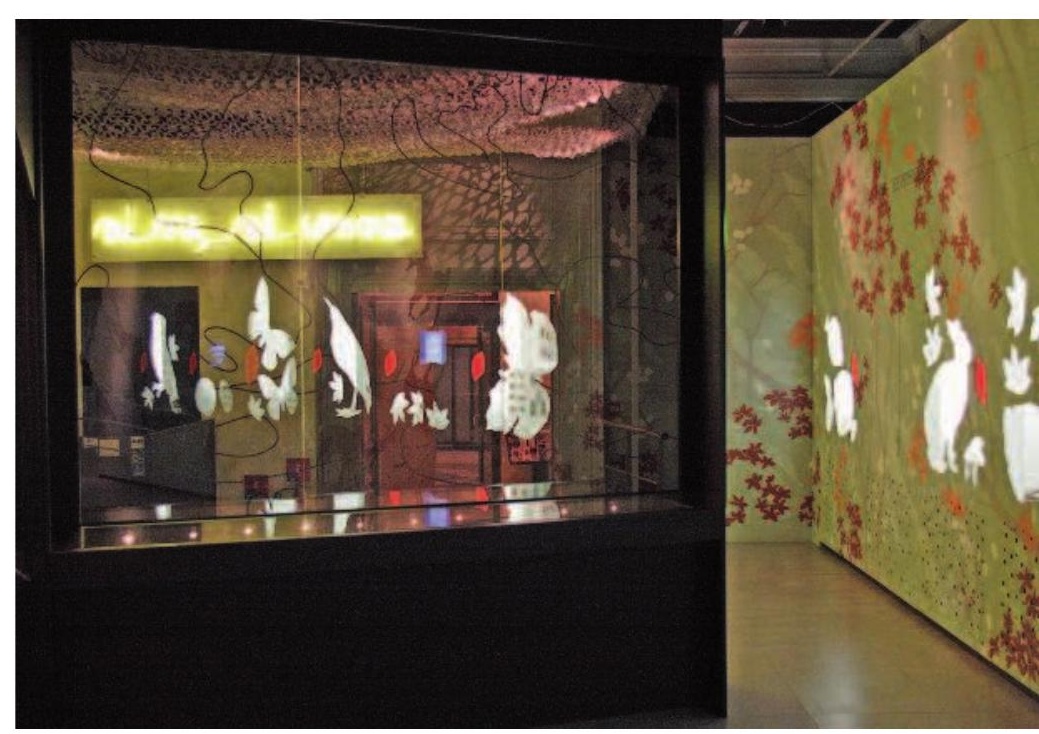

L'apparition du titre de l'exposition ๑) Muséum-Département du Rhône/Patrick Ageneau 


\section{Des objectifs de contenu}

Le parcours proposé est thématique et didactique. Il s'articule autour de six thèmes successifs : le « camouflage décamouflé », "fusionner et sociabiliser, une affaire de je(u)x de rôles », « le temps du secret », le « camouflage malgré soi », la «transparence » et le « camouflage jusqu'à l'extrême : l'invisibilité ».

L'exposition débute par une partie introductive et d'interpellation. Le public y découvre ce qu'il connaît et associe généralement au camouflage. Cette mise en scène du camouflage évident, du stéréotype, est une mise en condition qui, très vite, amène à se questionner sur l'existence d'autres formes de camouflage. Tout au long de l'exposition le visiteur avance ainsi dans une exploration de camouflages inattendus, de plus en plus subtils, pour enfin aboutir au fantasme humain du camouflage ultime : l'invisibilité matérielle jusqu’à la dématérialisation de l'être.

Le principal objectif de l'exposition consiste à amener le public à se questionner sur les réels mécanismes des camouflages mis en œuvre dans la nature, à faire déceler le camouflage là où on ne l'attend pas, notamment dans les relations humaines, interpersonnelles et intra-personnelles. Enfin, il s'agit aussi de faire découvrir les multiples fonctions de la dissimulation et de la révélation par l'expérimentation et l'implication émotionnelle.

L'exposition prend donc à contre-pied certaines idées reçues attachées au camouflage. Ainsi, on s'aperçoit que les fonctions du tissu « camouflage », ou «motif bariolé » militaire, ne se limitent pas à la dissimulation. On découvre aussi que certains phénomènes de camouflages animaliers tiennent plus de la projection de nos perceptions humaines sur la nature que d'intentions de camouflages. En explorant ces a priori, l'exposition permet de recentrer la réflexion sur l'être humain. Il s'agit alors de considérer l'individu en tant que producteur et utilisateur de multiples camouflages dans la société.

\section{Une scénographie de camouflages}

Ni vu, ni conmu s'inscrit dans la programmation des expositions thématiques du muséum en traitant un sujet de manière transdisciplinaire. Sa particularité est d'offrir une place prédominante à la scénographie qui soutient paradoxalement de manière visible un discours de la dissimulation. L'exposition expérimente délibérément une étroite imbrication de la scénographie au contenu qu'elle théâtralise au service de son propos et de sa compréhension.
Ni vu, ni connu est une exposition de discours. Dans cette démarche, l'objet se met au service de celui-ci et le soutient. D'ailleurs, le mode de hiérarchisation des cartels situe d'abord l'objet dans le propos de l'exposition avant de le documenter plus précisément en développant ses caractéristiques propres. Pour cette raison, un travail important a été d'élaborer des contenus et de scénariser le discours, préalablement à la sélection des objets. Cette démarche permet ainsi de confronter conjointement le public à une grande variété d'objets, de statuts différents, autour d'un même sujet : collections d'Histoire naturelle, collections ethnologiques, oeuvres classiques et d'art contemporain, reconstitutions, artefacts, dispositifs visuels et sonores...

La mise en scène privilégie l'utilisation de dispositifs participatifs distincts, divers et variés, des espaces d'immersion, d'interpellation ou de déstabilisation. La scénographie joue alors avec les visiteurs, qui eux-mêmes jouent avec les dispositifs. Nous avons sollicité différents modes de perception du propos

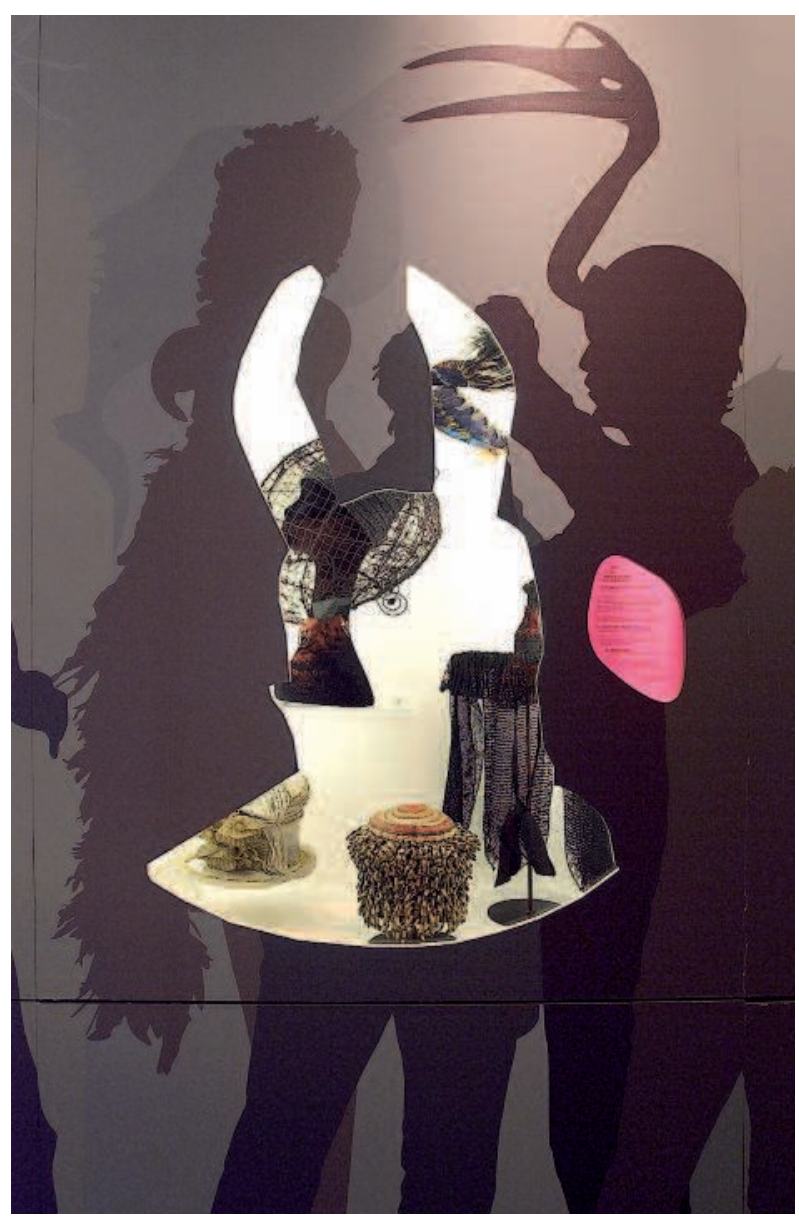

Des cartels groupés de couleur rouge comme fil conducteur du discours ๑) Muséum-Département du Rhône/Bruno Lapray 


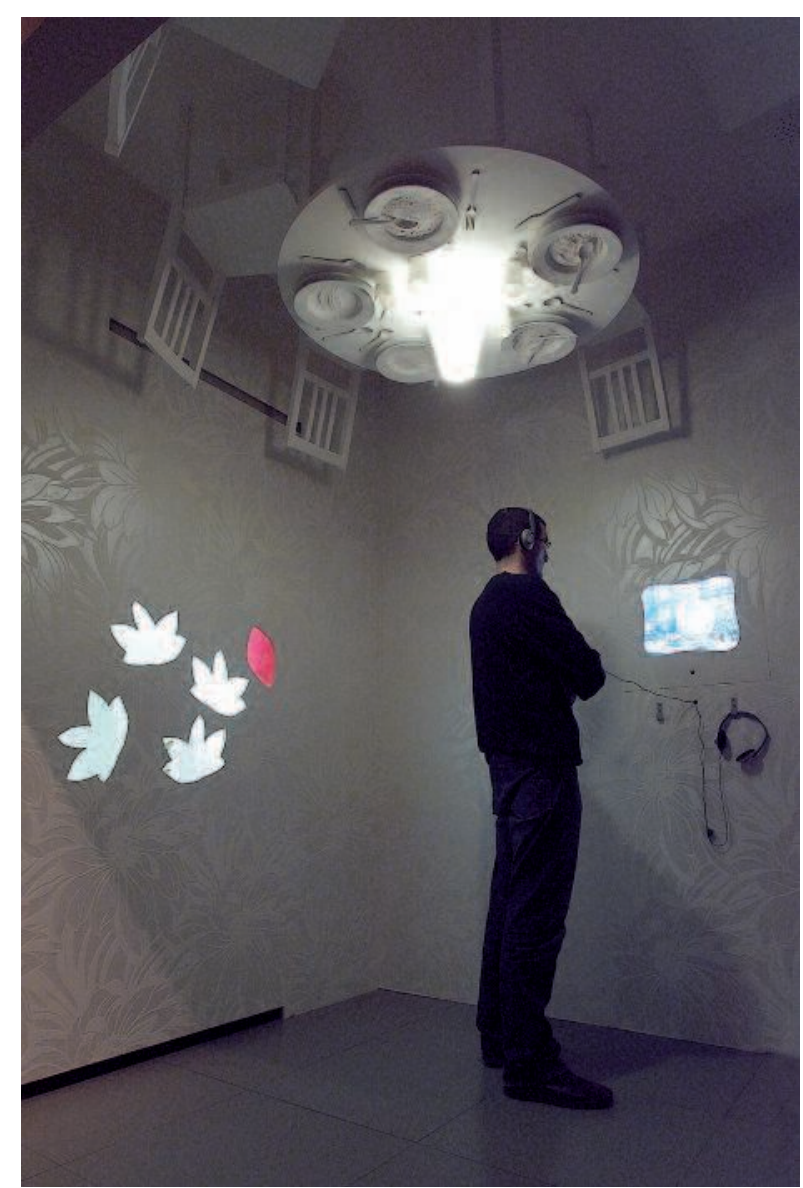

Changement de perspective avec ce décor suspendu à l'envers au plafond ○ Muséum-Département du Rhône/Bruno Lapray

parfois même à travers des niveaux de réception euxmêmes distincts et donc une variété de modes d'appropriation de l'exposition, essayant en cela de répondre aux différents formats de visite des publics. Jouant ainsi à la fois sur sa dimension esthétique, immersive, ludique, corporelle et se voulant riche en expériences pour le public. Au visiteur de choisir d'activer tout ou partie de celles-ci à des niveaux ou sur des registres différents (objets de collections, graphisme, lumière, interactifs, sons, images animées, textes...) et de construire au fur et à mesure son propre sens du discours.

Pour cela, le parti pris muséographique est fort. La mise en scène choisie se veut spectaculaire et théâtrale jusqu'à solliciter corporellement le public afin de privilégier l'expérimentation de ses questionnements. La proposition de réelles expériences de camouflage relaie le propos de l'exposition dans des jeux d'opacité, de transparence, d'invisibilité, de masques et de dévoilements, d'ombres et de mises en lumière. Et il semble que l'implication corporelle du visiteur souhaitée dans le projet et mise en place par la scénographie de Ni vu, ni connu participe bien à la construction du sens (2).

Il s'agit alors de suggérer le camouflage, notamment au moyen de fenêtres sur les vitrines dont les découpes représentent des silhouettes en lien avec le thème et les objets présentés. Ce masquage invite le visiteur à un jeu de regards et complète le propos de l'exposition. Pour voir les animaux en posture de camouflage, il doit les rechercher et pour cela bouger son corps de la même façon qu'il le ferait en pleine nature. Le titre de l'exposition devient intelligible lorsque le visiteur, lui-même camouflé derrière une glace sans tain, le découvre à l'endroit et par transparence ; la temporisation de l'éclairage de vitrines cache et révèle certains objets ; un capteur de rêves est présenté sous l'apparence de son ombre portée ; un décor mis à l'envers au plafond provoque un changement de perspective ; le visiteur est immergé dans le rêve énigmatique d'un autre projeté sur grand écran. Il découvre dans un premier temps l'image par l'envers de l'écran puis le contourne et décrypte le rêve grâce aux sous-titres...

Certains effets recherchés sont même conçus pour fonctionner de manière implicite. Le visiteur découvre, grâce à une borne interactive « Directiv Vision » (3), le changement d'identité qui s'opère lorsque l'on met un masque. La position d'un miroir derrière la présentation de masques en rapport avec la borne permet au visiteur de voir inconsciemment son propre visage noyé au milieu d'une foule imprimée en filigrane sur un rideau incurvé situé derrière lui. Une expérience d’invisibilité est également proposée au visiteur, invité à se revêtir d'une cape et découvrant,

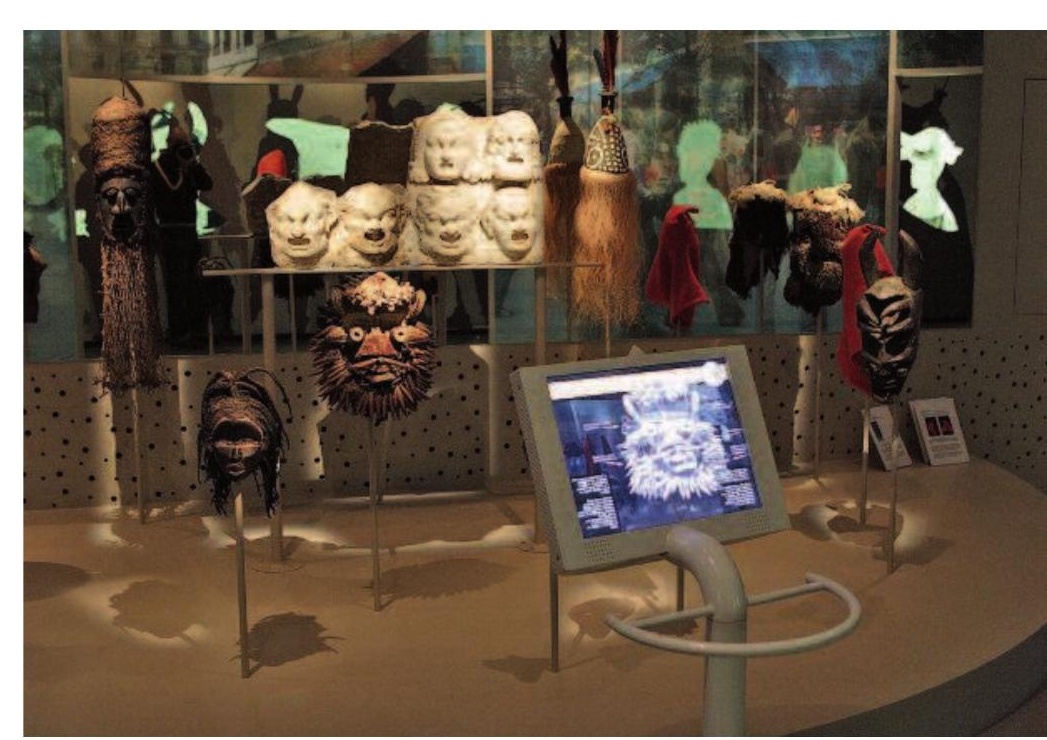

Les masques et la borne interactive Directiv Vision

(C) Muséum-Département du Rhône/Patrick Ageneau 
dans un moment de flottement, la projection décalée dans le temps de son image ou seule sa tête demeure visible. Le trouble qui s'en suit lui suggérant que l'invisibilité n'est pas une expérience sans risque.

La scénographie de l'exposition constitue donc un support de signification essentiel de son propos, au point que certains visiteurs vont même jusqu'à la considérer comme objet ${ }^{(4)}$.

\section{Une position atypique du média texte}

Parallèlement à cette position de la scénographie, l'exposition se distingue par une faible présence du texte écrit. L'absence de texte introductif, de textes de zones, de cartels essentiellement descriptifs, laisse place à d'autres formes textuelles, sonores, soustitrages à l'écran, intégration au graphisme.

Parmi tous les jeux de dissimulation graphique, les cartels, eux, détonnent. Leur ensemble constitue un fil rouge qui ponctue le mobilier d'exposition et vient équilibrer les différences de mise en scène du sujet. Communs à plusieurs objets illustrant un même sous-thème, leur conception privilégie un premier niveau de texte court, en gros caractères, lié essentiellement au discours principal, et adapté à une prise d'information légère qui fait d'autant mieux sens. Elle invite à la contemplation de ce qui devient alors important, les objets, et à l'expérience sensible qu'ils font vivre sans le filtre d'un lecture informative. Le texte lié à la description de l'objet ne vient qu'en second temps puisqu'il n'est pas primordial à la bonne compréhension du propos.

Le graphisme même des textes, appuyé par les autres médias (lumières, sonorités...), étant adapté à chaque zone, il en soutient le sens et permet au public d'en comprendre la thématique sans l'obliger à une lecture exhaustive. Nous avions pour objectif l'idée que la muséographie prenne en charge ce que nous ne voulions pas faire avec le texte « classique ». Le but était de faire du texte un média graphiquement intégré à la scénographie, le faire participer de l'ambiance muséographique et privilégier un rapport sensible au propos ${ }^{(5)}$. De manière révélatrice, la scénographe, Nathalie Crinière, qualifie même le résultat de cette intégration de «scénographisme» (6).

\section{L'expérimentation des puces RFID dans l'exposition}

Nous nous devions d'aborder, dans une exposition traitant du thème du camouflage, l'un des fantasmes récurrents de la littérature d'anticipation qui trouve de plus en plus d'échos dans l'actualité et que l'on dénomme le plus souvent «Big Brother » en référence au célèbre roman 1984 de George Orwell. L'auteur y décrit une société sous contrôle permanent du pouvoir, où toute liberté d'expression a disparu grâce à la surveillance et au contrôle de l'ensemble des pensées des citoyens.

Il nous importait de montrer le fonctionnement et l'utilisation accrue dans notre vie quotidienne de certaines technologies de l'information dites «pervasives » (omniprésentes). En effet, en recueillant et en stockant furtivement, voire abusivement, des informations sur nos déplacements, nos types d'achats..., ces technologies peuvent comporter un risque pour la protection de la vie privée et des libertés individuelles.

Nous souhaitions que le visiteur vive une véritable expérience de "surveillance » sans y être préparé pour que la surprise et la réaction provoquées au moment de sa découverte lui permettent une réflexion d'ordre éthique sur une problématique sociale et politique contemporaine : quelles peuvent être les conséquences possibles de l'utilisation des

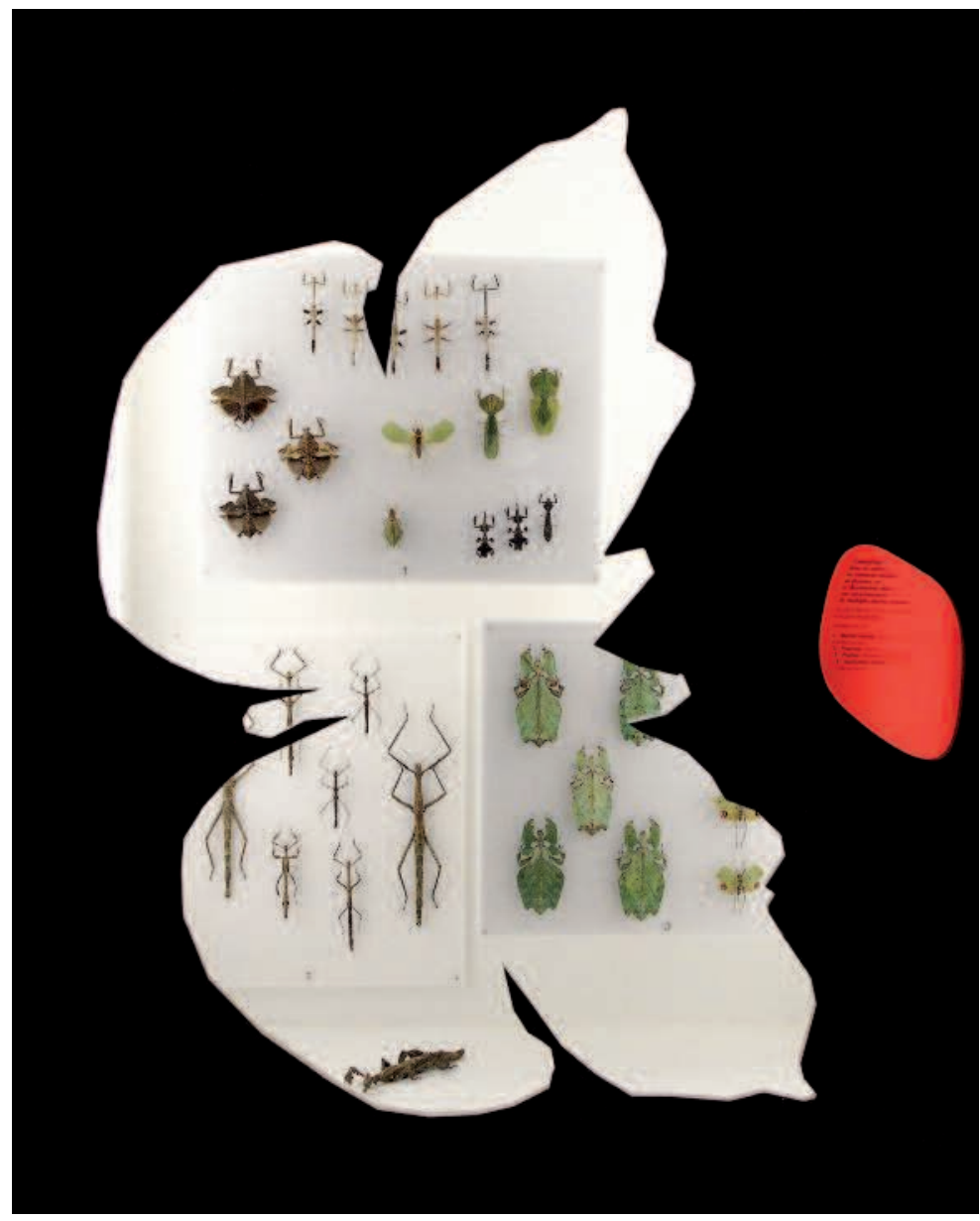

Les visiteurs découvrent les objets à travers les découpes des vitrines. (c) Muséum-Département du Rhône/Bruno Lapray. 
technologies capables de détecter, stocker et transmettre des informations personnelles et de notre vie quotidienne?

Le système retenu pour mettre en place ce scénario, l'utilisation de puces RFID, était donc pour nous avant tout le moyen d'une médiation participative, impliquante, ludique et réflexive. Secondairement ce choix nous offrait également la possibilité de récolter un certain nombre d'informations sur l'ensemble du parcours des visiteurs dans l'exposition, l'usage, l'ergonomie et la fiabilité d'interactifs et de ce système d'identification automatique par radiofréquence intégré dans une muséographie.

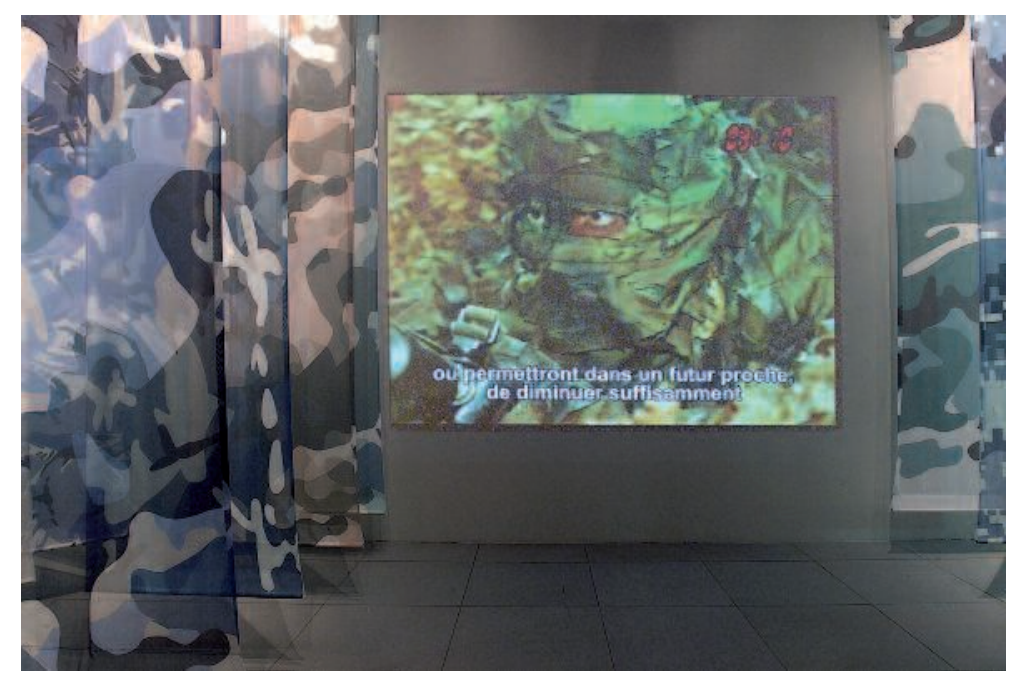

Le scénario « RFID » était donc inclus au propre scénario de l'exposition et décomposé en quatre étapes. Techniquement, il reposait sur le principe de camoufler une puce RFID dans une carte identifiée remise au visiteur dans le cadre d'un jeu-énigme et de collecter à son insu un certain nombre de renseignements (7). Il était suivi, identifié, reconnu, photographié, puis à la sortie du parcours sa photographie et l'identité qu'il nous avait fournie étaient affichées à la «Une » d'un journal «people » fictif qui lui était uniquement destiné. S'ensuivaient alors une explication du procédé utilisé dans l'exposition, une information sur l'utilisation des systèmes d'identification automatique par radiofréquence et une communication sur le rôle de la Commission nationale de l'Informatique et des Libertés (CNIL) et sur les droits et protections du citoyen. Le visiteur avait alors le choix de détruire les informations le concernant ou bien de se les faire envoyer à son adresse courriel. Elles étaient accompagnées de la "Une » du journal «people » et d'une information sur le rôle et le fonctionnement de la CNIL.

\section{Conclusion}

L'objectif principal de cette exposition est de concevoir l'espace comme média intégré du contenu. La scénographie sollicite non seulement le regard du visiteur, son écoute, sa participation, mais aussi sa position corporelle afin de le mettre en situation d'expérimenter réellement des types de camouflages. La scénographie joue également un rôle de liant entre les différents registres composant $\mathrm{Ni} v u, n i$ connu. Enfin, elle participe pleinement à la perception de l'exposition conçue comme un expérience globale à vivre.

La prise en charge du fond par la forme et un brouillage de la délimitation entre l'un et l'autre
Graphismes de faux motifs de camouflages militaires imprimés sur des voilages : l'ensemble des audiovisuels de l'exposition sont sous-titrés. ๑) Muséum-Département du Rhône/Bruno Lapray

n'ont pas empêché l'exposition de fonctionner, bien au contraire. Ainsi, le graphisme explicite, donne des repères, il participe au récit et à sa mise en forme en jouant le jeu des camouflages.

L'expérimentation d'un système d'identification automatique par radiofréquence nous a permis de traiter une thématique et sa médiation de manière originale et surprenante dans une véritable expérience impliquante pour le public. De plus, les informations qu'elle nous a permis de recueillir sur les pratiques du parcours de l'exposition par les visiteurs et leur usage d'interactifs et du système RFID lui même se sont révélées riches d'enseignements qui servent notre réflexion générale dans la perspective d'éventuelles applications au futur musée des Confluences.

\section{Notes}

(1) L'exposition, présentée sur une superficie de $650 \mathrm{~m}^{2}$ du 8 novembre 2005 au 2 juillet 2006, a accueilli près de 40000 visiteurs. Destinée à un public familial, une attention particulière a été portée au public malentendant (sous-titrage adapté de l'ensemble des audiovisuels).

(2) Candito, N. Expérience de visite et dispositifs participatifs. La place du corps dans la perception du propos de l'exposition et Miège, D. Évaluation, développement et stratégie, actes des journées du Louvre $1^{\text {er }}$ et 2 juin 2006, p. 11 (à paraître à La Documentation française).

(3) Borne Interactive Multimédia Orientable dite de « réalité augmentée » permettant au visiteur de sélectionner un objet de collection à informer en dirigeant le dispositif sur lui.

(4) Miège, D. Les influences d'une muséographie de la sensation sur la

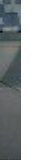


réception des publics : diversité accrue des modes d'appropriation de l'exposition. Évaluation qualitative de l'exposition «Ni vu ni connu - Paraître, disparaître, apparaître », Avignon : Laboratoire Culture et Communication, 2006, p. 53.

(5) Ibid, p. 6

(6) Texte de présentation des intentions scénographiques (Agence NC, scénographe de l'exposition)

(7) Conformément à la législation en vigueur, cette collecte a été déclarée auprès de la CNIL L’ensemble du projet a été réalisé sous son contrôle et en collaboration avec A.-M. Benoit.

\section{Bibliographie}

Bureau, S. L'objet et les technologies de l'information et des communications, rapport de stage, cellule évaluation du muséum, université Jean Moulin Lyon 3, août 2006, 87 p.

Chabert, V. Ni vu ni connu : camouflages. Lyon : Muséum, Biro éditeur, 2005,234 p.
Candito, N.et Miège, D. Expérience de visite et dispositifs participatifs. La place $d u$ corps dans la perception du propos de l'exposition. Lyon : Muséum et Avignon : Laboratoire Culture et Communication, actes des journées du Louvre 1-2 juin 2006, 13 p.

Hervy, E. Camouflage, Étape graphique, mai 2006, pp. 69-72.

Miège, D. Les influences d'une muséographie de la sensation sur la réception des publics : diversité accrue des modes d'appropriation de l'exposition. Évaluation qualitative de l'exposition «Ni vu ni connu - Paraître, disparâิtre, apparaître », Laboratoire Culture et Communication, Avignon, 2006, $79 \mathrm{p}$.

Site de l'exposition $\mathrm{Ni} v u$, ni connu : www.museum-lyon.org/expo_temporaires/ni_vu_ni_connu/

Site du collectif artistique interdisciplinaire la Mercerie, Michel Jeannès. : www.lamercerie.eu/ 